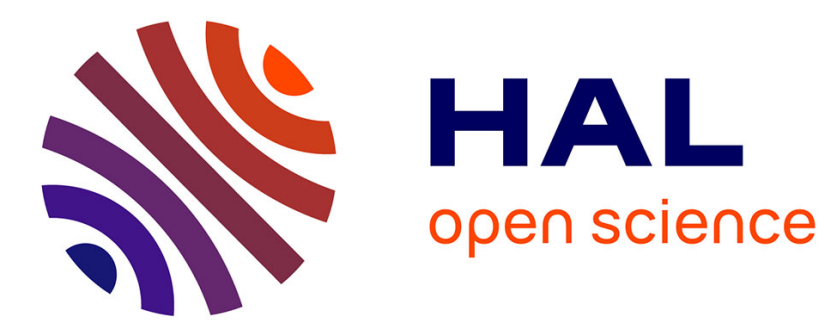

\title{
REAL TIME FOURIER TRANSFORM SYNTHESIZER
}

Jakobus Hoffman, G. Vanasse

\section{To cite this version:}

Jakobus Hoffman, G. Vanasse. REAL TIME FOURIER TRANSFORM SYNTHESIZER. Journal de Physique Colloques, 1967, 28 (C2), pp.C2-79-C2-85. 10.1051/jphyscol:1967216 . jpa-00213194

\section{HAL Id: jpa-00213194 https://hal.science/jpa-00213194}

Submitted on 1 Jan 1967

HAL is a multi-disciplinary open access archive for the deposit and dissemination of scientific research documents, whether they are published or not. The documents may come from teaching and research institutions in France or abroad, or from public or private research centers.
L'archive ouverte pluridisciplinaire HAL, est destinée au dépôt et à la diffusion de documents scientifiques de niveau recherche, publiés ou non, émanant des établissements d'enseignement et de recherche français ou étrangers, des laboratoires publics ou privés. 


\title{
REAL TIME FOURIER TRANSFORM SYNTHESIZER (*)
}

\author{
J. E. HofFman \\ Concord Radiance Laboratory \\ Division of Utah State University Davis Road \\ Bedford, Massachusetts, U. S. A. \\ and \\ G. A. VANASSE \\ Optical Physics Laboratory \\ Air Force Cambridge Research Laboratories (O. A. R.) \\ Laurence G. Hanscom Field Bedford, Massachusetts, U. S. A.
}

\begin{abstract}
Résumé. - Nous décrivons une technique de synthèse en temps réel du spectre à partir de l'interférogramme correspondant. La synthèse spectrale est accomplie par des sommes successives de fonctions sinusoïdales dont les amplitudes sont déterminées par les points d'échantillonnage de l'interférogramme. La fréquence de ces fonctions est fixée par le signal de référence. La somme des fonctions sinusoïdales est effectuée dans la mémoire digitale à 1024 adresses d'un oscilloscope. Le système qui sert à déterminer le point de différence de marche zéro est décrit, ainsi que le fonctionnement et les performances du synthétizeur de Fourier en temps réel ; des exemples de résultats sont aussi présentés.
\end{abstract}

Abstract. - A real time technique to synthesize the spectral distribution from the corresponding interferogram is described. The spectral synthesis is performed by successive summation of cosine functions with amplitudes which are determined by the sample points of the interferogram. The frequency of these functions is controlled by the reference signal. Summation of the cosine functions is performed in a digital memory oscilloscope which has 1024 separate addresses. The system to determine the point of zero path difference is described. The operation and performance of the real time Fourier synthesizer are described and samples of the data obtained are presented.

The interferogram $F(x)$, corresponding to the spectral distribution $E(v)$, is the output of an interferometer used for Fourier spectroscopy. The interferogram is represented by

$$
F(x)=\int_{v_{m}}^{v_{M}} E(v) \cos 2 \pi v x \mathrm{~d} v,
$$

where $v_{m}$ and $v_{M}$ are the minimum and maximum wavenumbers in the spectral distribution. From equation (1), we see that the interferometer has synthesized the interferogram by the superposition of a series of trigonometric functions with amplitudes determined by the spectral function.

(*) This work was supported in part by the Laboratory Directors Fund, AFCRL, and by contract AF-19 (628)-251.
The spectral distribution is related to the interferogram by

$$
E(v)=\int_{-\infty}^{\infty} F(x) \cos 2 \pi v x \mathrm{~d} x .
$$

It is reasonable, then, to obtain the spectral distribution by the inverse process, i. e., by a superposition of trigonometric functions with amplitudes determined by the interferogram. A synthesis of the spectral function is thus obtained and this synthesis is the Fourier series approximation to the spectrum.

The interferogram function is sampled according to the requirements of the sampling theorems and equation (2) becomes

$$
E(v)=\sum_{n=-\infty}^{\infty} F\left(x_{n h}\right) \cos 2 \pi v x_{n h},
$$


where $F\left(x_{n h}\right)$ is the value of the interferogram at the sample point $n h$ and $h$ is the sampling interval.

The interferogram is truncated and is assumed to be an even function. Under these conditions, equation (3) may be rewritten as

$$
E(v)=\sum_{n=0}^{N} F\left(x_{n h}\right) \cos 2 \pi v x_{n h},
$$

where $N$ corresponds to the last sample point or to the maximum path difference.

The spectral synthesis is represented by

$$
\varphi(\omega)=\sum_{n=0}^{N} G\left(t_{n h}\right) \cos \omega_{n} t
$$

where $\varphi(\omega)$ is the spectrum of the detector response and $G\left(t_{n h}\right)$ is the amplitude of the detector response at the sample point $n h$. The angular frequency of the cosine functions is given by

$$
\omega_{n}=2 \pi f_{n}=2 \pi(n / 2 \Delta v)
$$

where $\Delta v$ is the bandwidth of the incident radiation and the running variable $t$ is equivalent to the parameter $v$. Each sample point of the interferogram is used to generate a cosine function with an amplitude determined by the sample value and a frequency determined by the path difference.

Equation (5) forms the basis of the real-time Fourier synthesizer. The sample value $G\left(t_{n h}\right)$ is held while it is multiplied by the proper cosine function. Each succeeding product is put into the memory and added to the preceding data. A summation of $G\left(t_{n h}\right) \cos \omega_{n} t$ terms is thus obtained.

A block diagram of the real-time Fourier transform synthesizer is shown in figure 1 . The instrument has been designed to operate with a lamellar grating interferometer but it could also be used with a wellcompensated Michelson.

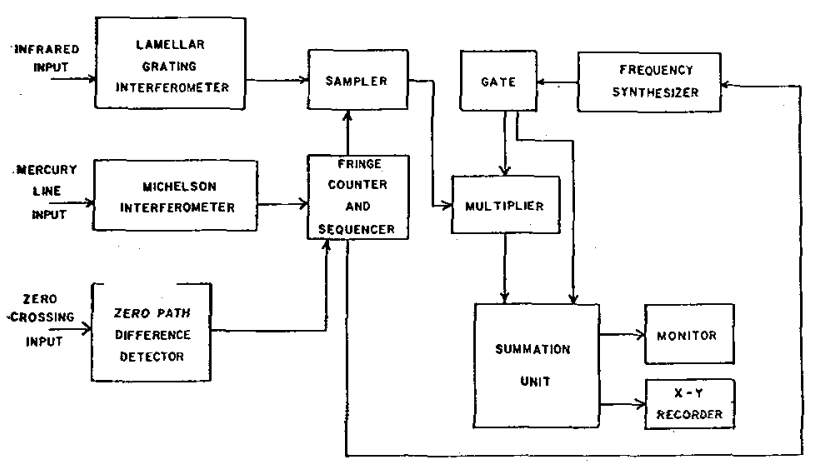

Fig. 1. - Basic block diagram of the real-time Fourier synthesizer.
Four signals are required to synthesize the spectral distribution. Three of these are inputs to the instrument while the fourth is internally generated. The inputs are : the output of the lamellar grating interferometer, the reference signal and the pulse which is derived from the zero path difference detector. This pulse initiates the automatic operation of the instrument. The reference signal is derived from a Michelson interferometer, the moving mirror of which is mounted on the rear of the moving facet of the lamellar grating.

In order to synthesize the cosine transform of the interferogram, it is necessary to know when the path difference between the two beams is zero. This information is supplied by the zero path difference detector, which will be described later.

Signals go through the instrument along one of several paths. The output of the interferometer traverses the first path, which extends from the detector of the interferometer through several stages of amplification, through an analog sample and hold circuit and ends in the multiplier. The interferogram signal is sampled, and $G\left(t_{n h}\right)$ terms are produced. The sample value is held during the interval between samples and it is during this interval that it is multiplied with the proper cosine function.

The cosine functions, required for the synthesis, are generated in the second signal path. This extend from the frequency synthesizer through the gate circuit and ends in the multiplier. The frequency synthesizer generates a sinusoidal signal with a precisely known frequency. All the frequencies are derived from a crystal controlled master oscillator. The frequency synthesizer is remotely controlled and the frequency of the output can be changed on command. The reference signal changes the frequency according to a pre-set program.

A gate circuit and its associated control circuitry are used to obtain cosine functions from the sinusoidal signal generated by the frequency synthesizer. These cosine functions are then allowed to enter the multiplier.

The first two signal paths combine in an analog multiplier to form the third. Here, $G\left(t_{n h}\right)$ and $\cos \omega_{n} t$ terms are multiplied to form $G\left(t_{n h}\right) \cos \omega_{n} t$. These terms then go to a summation unit which consists of an analog to digital converter, a core memory with 1024 addresses and the required electronics.

The A-D converter is an integral part of the summation unit. It is accurate to within $0.5 \%$. It digitizes the incoming signal at 1024 ordinates and each of these values is stored in one address of the memory. 
Each time a new signal is stored, the old data is read out of each address, modified by the new and the result is re-stored in the same address. The memory is advanced from one address to the next by an external signal although it can also be done by an internal oscillator. New signals may be stored and summed at the rate of one each second. The summation unit will operate faster but the limitation is due to the allowable rate at which the interferogram may be sampled. The rate is governed by the characteristics of the scheme used to change the frequency of the frequency synthesizer and the frequency response of the summation unit.

When the mth sample is taken, $G\left(t_{m h}\right) \cos \omega_{m} t$ is produced in the multiplier. This is digitized and added to

$$
\sum_{n=1}^{n=m-1} G\left(t_{n h}\right) \cos \omega_{n} t
$$

which is already stored in the memory. After this signal has been stored, the memory contains

$$
\sum_{n=1}^{n=m} G\left(t_{n h}\right) \cos \omega_{n} t
$$

Corresponding ordinates of successive signals are stored in the same address.

Figure 2 shows several of the signals in the various paths. The input and output of the sample and hold circuit are shown, as are the first few cosine functions entering and leaving the multiplier.

The reference signal traverses the fourth signal

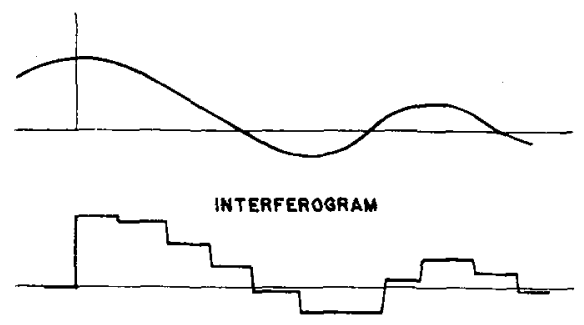

OUTPUT OF THE SAMPLE AND HOLD GIRCUIT
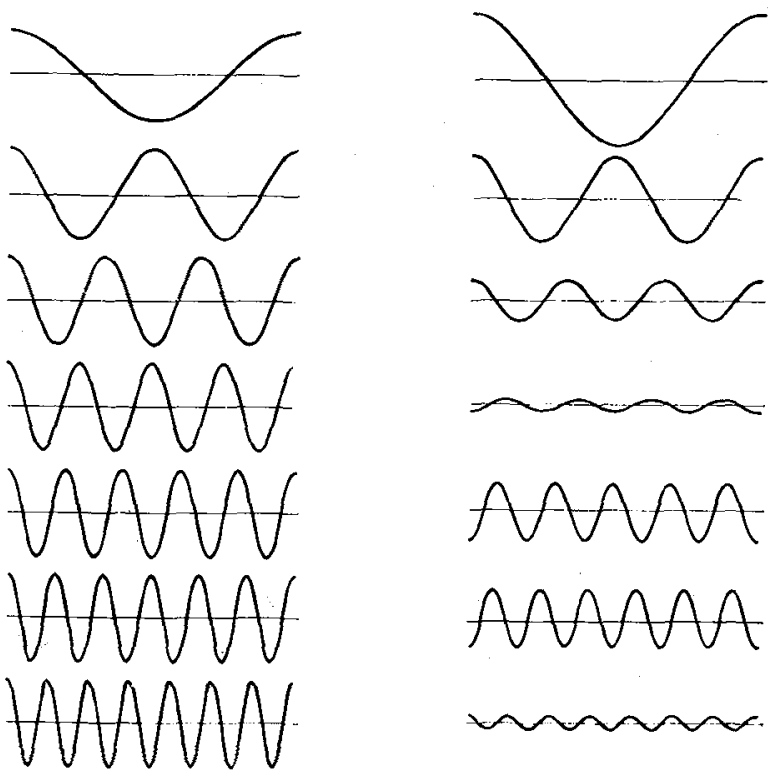

FIRST SEVEN FREQUENCIES ENTERING MUTIPLIER

FIRST SEVEN FREQENCIES ENTERING SUMMATION UNIT
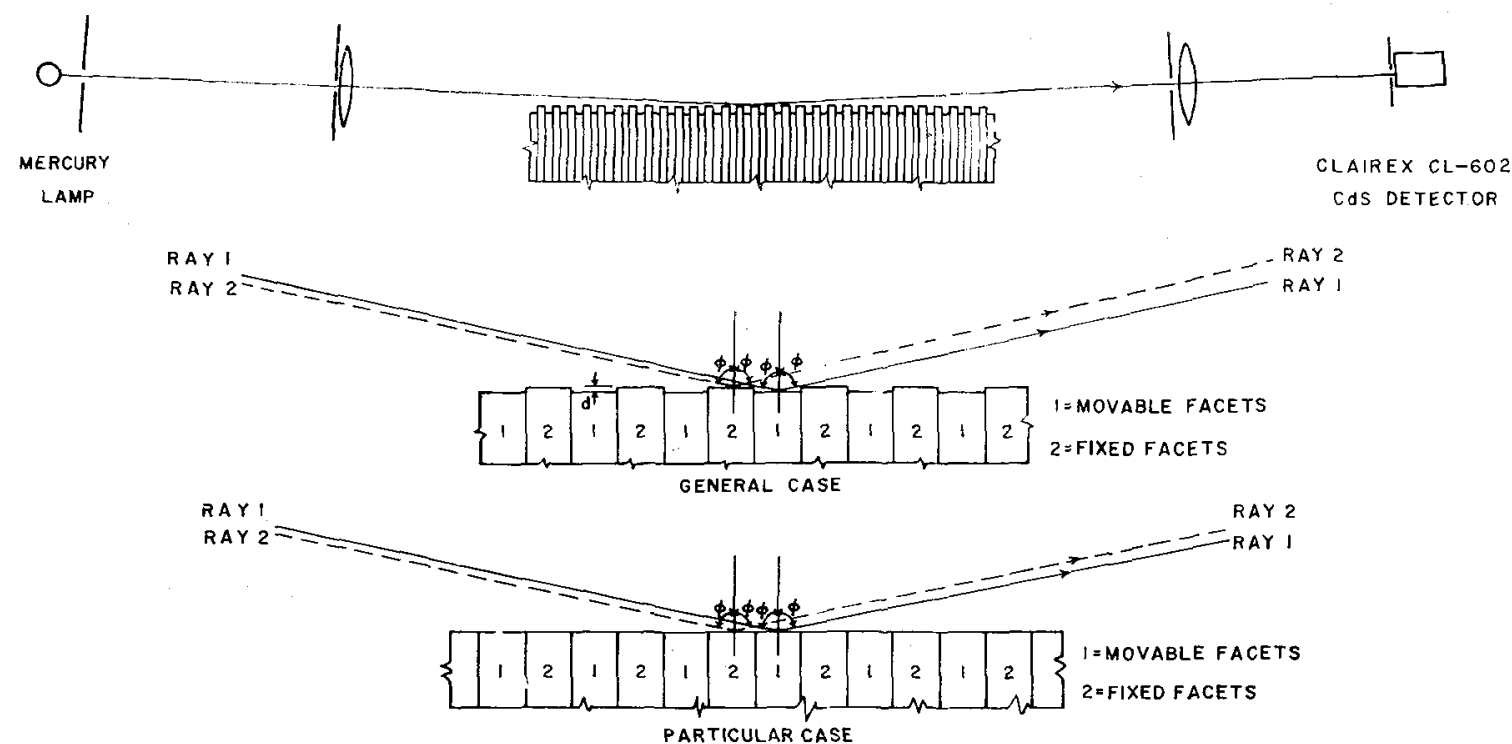

FIG. 3. - Optical system for the zero path detector. 
path. This splits into two branches. The first branch goes to the sample and hold circuit where it controls the sampling interval and the second branch goes to the sequencer which controls the frequency of the signal generated by the frequency synthesizer.

An oscilloscope monitors the data which are stored in the memory. The data may be read out with an $X-Y$ recorder as soon as the interferogram is complete.

The synthesized spectra repeat at intervals of $1 / h$, due to the sampling. The external advance signal is adjusted so the first 1000 addresses carry no repeating data.

Zero Path Difference Detector. - The zero path difference detector consists of an additional optical system and the associated electronics. Figure 3 shows a schematic of the optical system. The optical components are clamped to the frame of the lamellar grating. The lamp and collimator produce a very
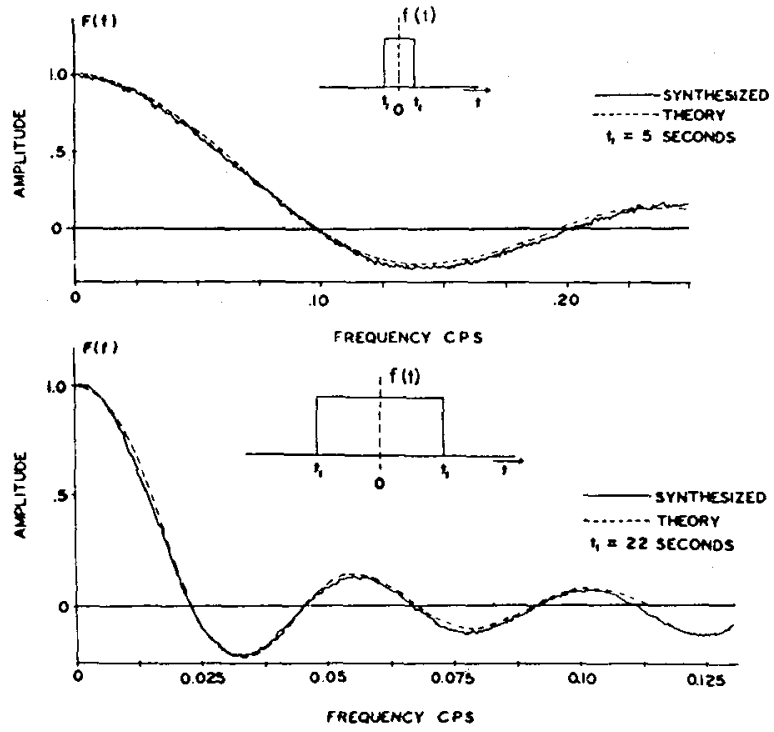

Frg. 4. - Theoretical and synthesized spectra for rectangular waveforms.
FIG. 5. - Synthesized spectra for truncated waveforms.
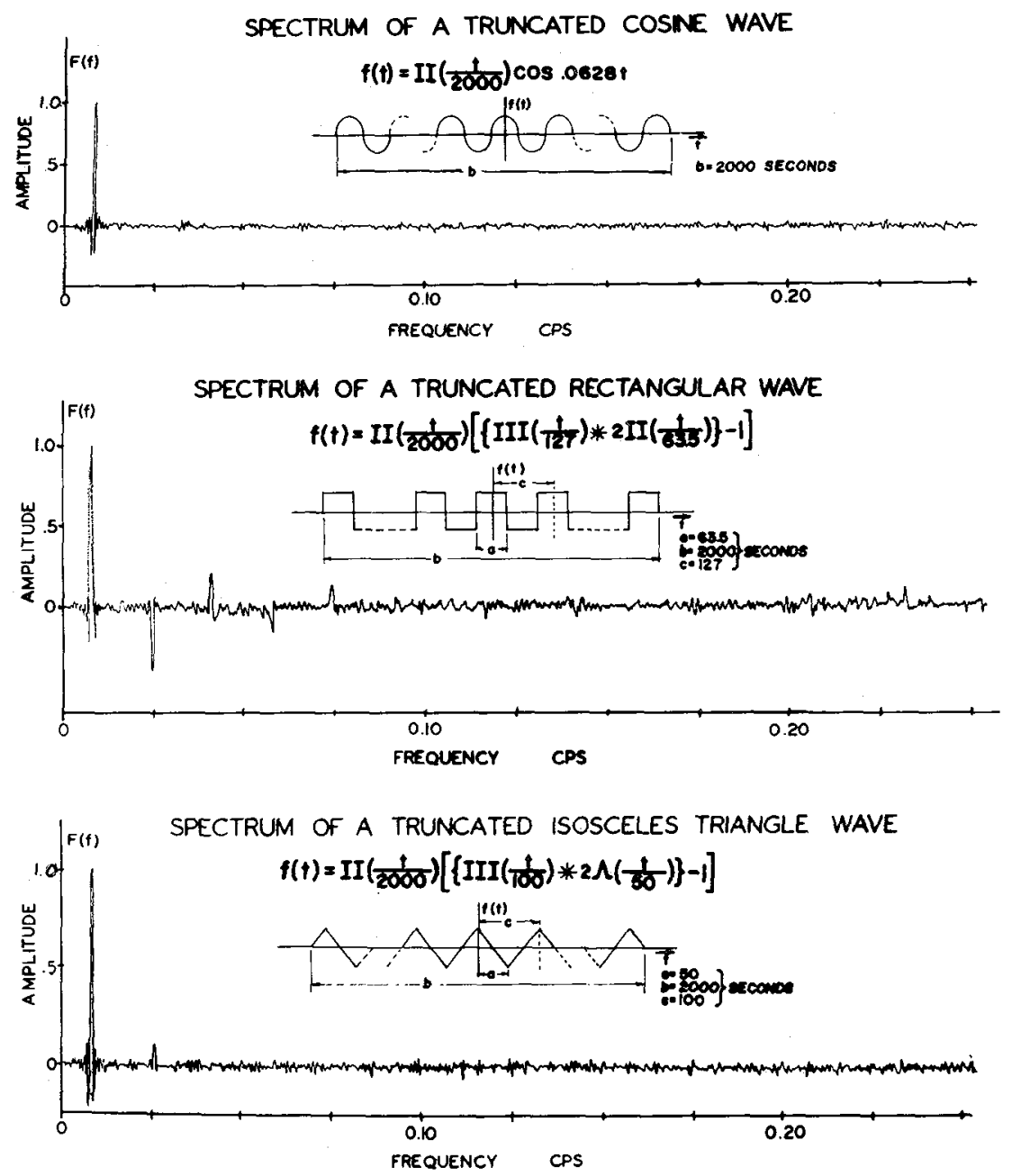


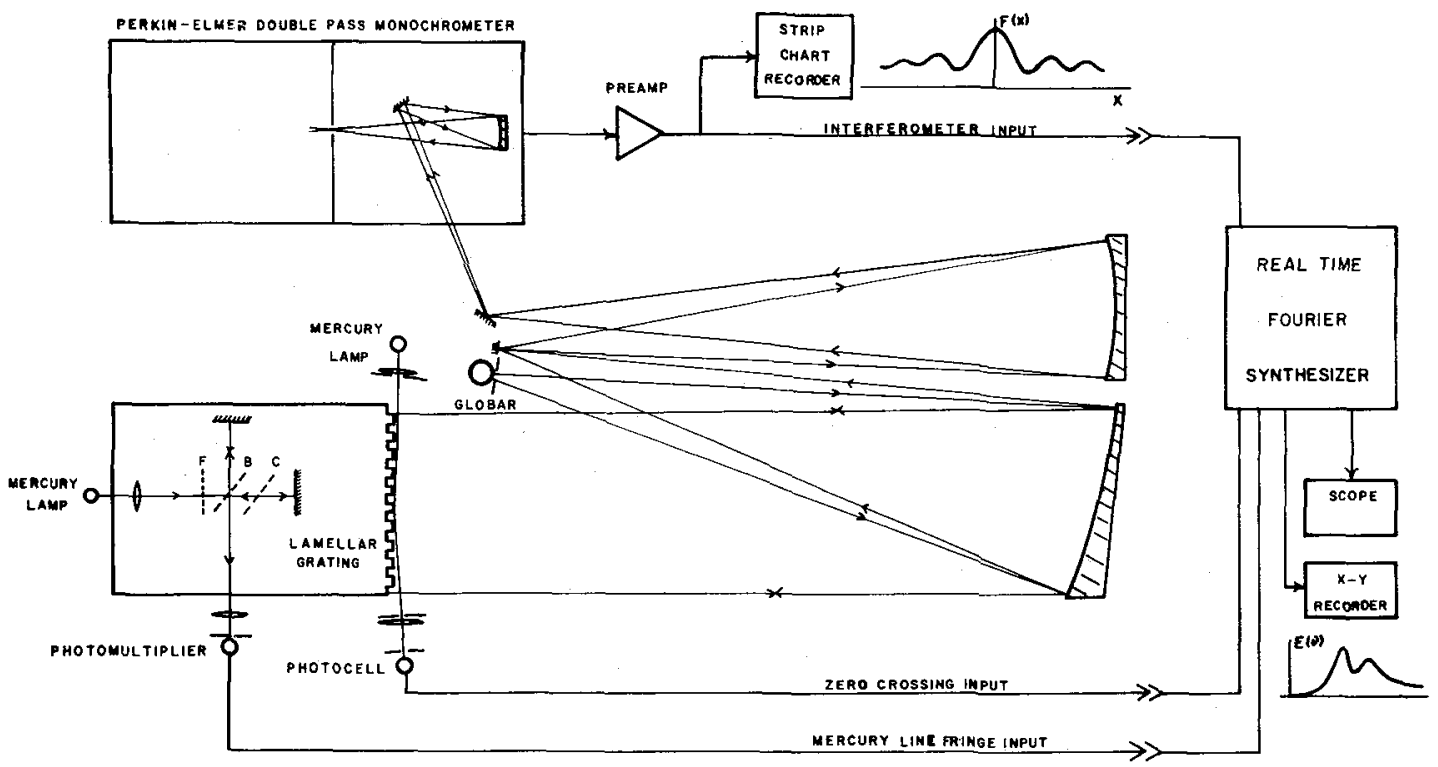

Fig. 6. - Experimental apparatus used to obtain the spectral distribution.

narrow beam of light which grazes the face of the grating and covers a narrow section of all the facets. Stops isolate the zeroth order interference pattern and a lens focusses it onto the detector. Figure 3 also shows the profile of the grating for the general case and for the case where the path difference is zero. In the latter case, the grating acts like a plane mirror and the detector response increases due to constructive interference. The angle of incidence is critical if a single humped response is to be obtained. An electronic circuit is used to generate a spike at the peak of the detector response. This spike initiates the sampling sequence.

The real-time Fourier transform synthesizer was evaluated using functions with known spectra. Figure 4 shows the synthesized and theoretical spectra obtained for rectangular functions while figure 5 shows the results for a series of truncated waveforms. The fluctuations are mostly due to digitization errors and recorder jitter.

Figure 6 is a diagram of the experimental apparatus used to obtain an interferogram and synthesize its corresponding spectrum. The monochromator serves as both an optical filter and a detector. Figure 7 shows the interferogram and the synthesized spectral distribution. The Fourier transform synthesizer is a real-time device and the synthesized spectrum builds up as more information is obtained. Figure 8 shows the same interferogram and the spectrum obtained at several points as the path difference is increased.

Figure 9 shows a photograph of the completed

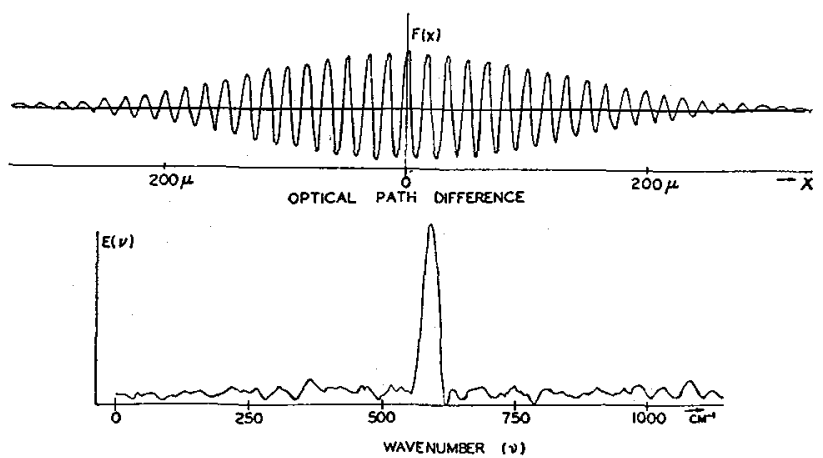

FIG. 7. - Interferogram and the Synthesized Spectral Distribution.
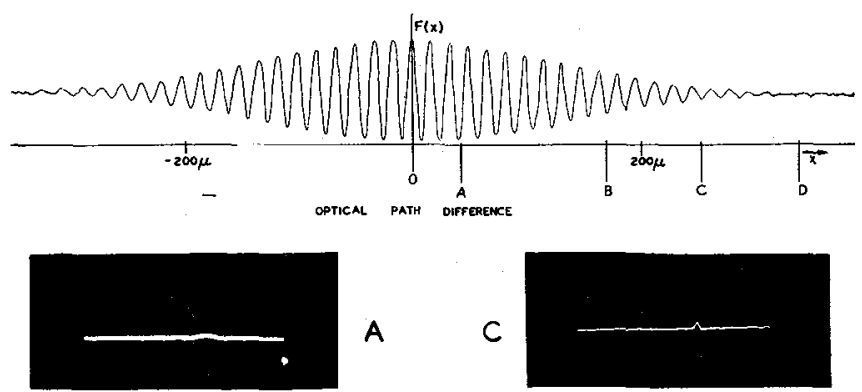

A

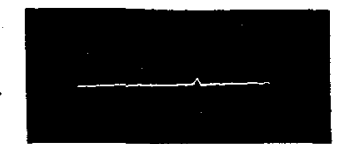

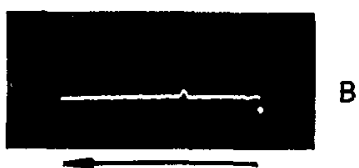

WAVENUMBER

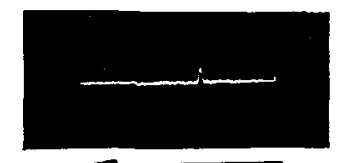

WAVENUMBER
FIG. 8. - Synthesized spectrum as a function of path difference. 

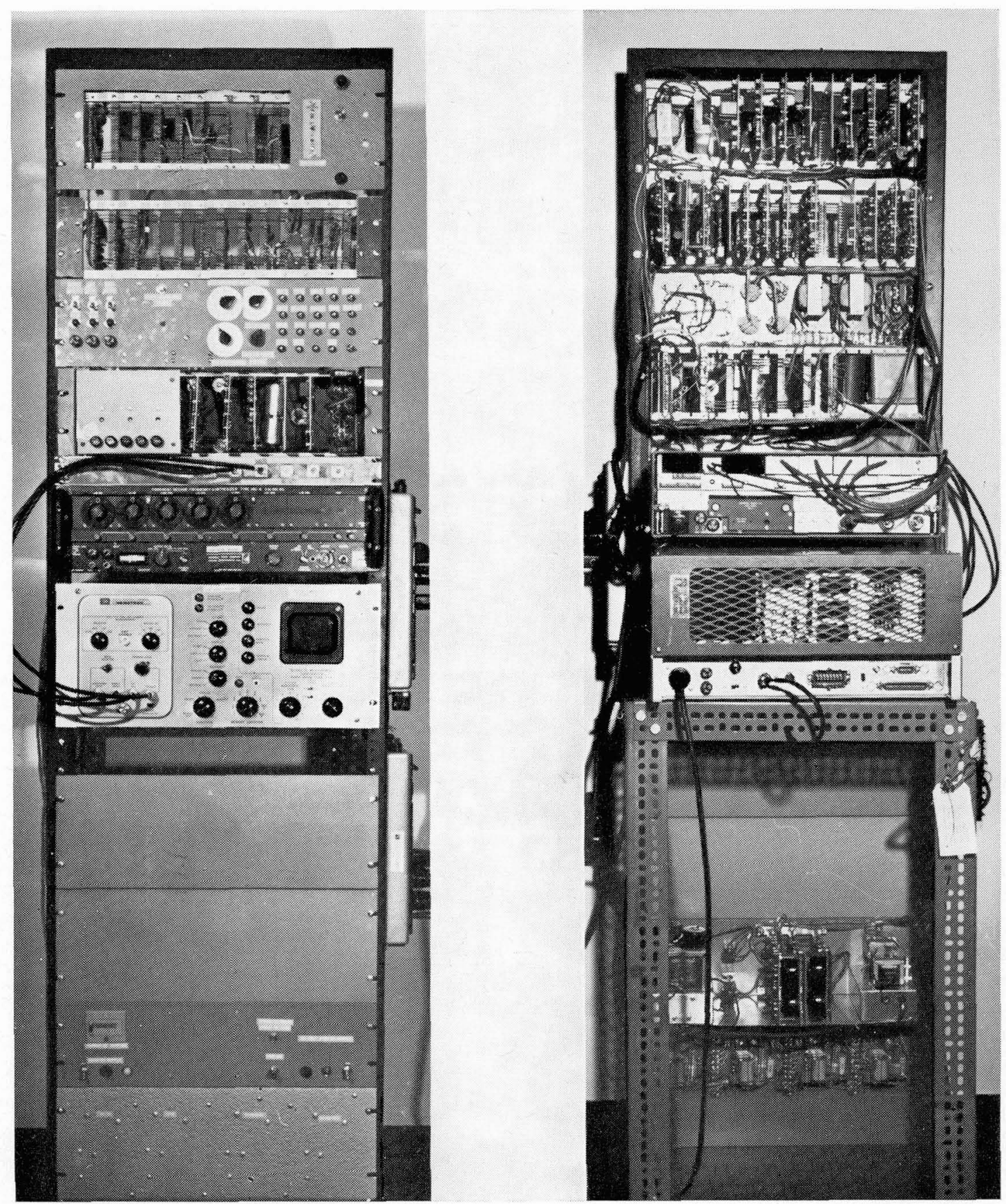

Fig. 9. - Photograph of the real-time Fourier transform synthesizer

real-time Fourier synthesizer. It is mounted in a single six-foot relay rack and occupies approximately three-quarters of it. The unit was designed so it could be moved from place to place. It is expected that some further work will be done which will enable it to be ruggedized and enable it to be used outside a la- boratory environment. It could be made considerably smaller by repackaging.

The present unit lacks an apodization system. This is expected to be added shortly. No great amount of work was done to optimize the zero path difference detector as greater interest was shown in perfecting 
the real-time techniques. Work will be done shortly to optimize the zero path difference detector. Work will also be done to reduce error to a minimum. Errors involved in synthesizing continuous spectra are presently about $2-5 \%$ with greater error occurring for broad band signals.

\section{INTERVENTIONS}

Note. - The following remarks were given during a discussion on the cost and usefullness of special purpose computers.

J. RiNG. - The cost of components in our instrument was about $£ 400$. The instrument is designed for use at sites remote from a computer (e. g. mountain observatories) or to make an immediate crude transform in the laboratory to check features of the source or satisfactory working of multiplex instruments. I believe that the majority of users of multiplex spectrometers do not yet have immediate access to a digital computer and will welcome a simple device which reduces computing costs by rejecting unsatisfactory interferograms.

J. L. Pritchard. - Not as much has been spent on our computer as might just be thought. Further these instruments [analog computers] are most useful as design tools for those of us who build interferometers.
I further doubt that we have wasted as much money on these computers as those who have used digital equipment very inefficiently because they didn't know how to program them. After all the information on how to program them has been available for a long time.

J. N. A. RIDYARD. - The cost of our analogue computer, amortised over ten years with a typical chemist's output of maybe ten spectra per working day, gives a spectral cost of less than one dollar for a double beam spectrum of up to a 200 to 300 resolving power. The spectrophotometer operator is the only operator necessary, and it is doubtful if even the Cooley program, if costed realistically, could approach this, for this type of low resolution spectra. The computer is available as a working system in a « black box ) which requires no further expenditure of time or money, apart from the moderate electricity supply.

The reliability of a purely electronic as opposed to mechanical systems involving tape transports need not be stressed.

The size of the unit is extremely compact, so the portability mentioned by the previous speakers is certainly very easy. There is only one cabinet of three feet by two feet by two feet, plus a small recorder case. This also includes the interferometer electronics. No worker need build a special laboratory to house this equipment. 